\title{
Concussion Education Programs for High School Football Coaches: An Overview
}

\author{
John Boudreaux, Jessica Dolecheck* \\ Department of Health Studies, College of Health Sciences, University of Louisiana Monroe, 700 University Avenue, Monroe, LA 71209 , United \\ States.
}

\section{Article Details \\ Article Type: Mini Review \\ Received date: $05^{\text {th }}$ December, 2018 \\ Accepted date: $20^{\text {th }}$ February, 2019 \\ Published date: $23^{\text {rd }}$ February, 2019}

"Corresponding Author: Jessica Dolecheck, Department of Health Studies, College of Health Sciences, University of Louisiana Monroe, 700 University Avenue, Monroe, LA 71209, United States. E-mail: dolecheck@ulm.edu

Citation: Boudreaux J, Dolecheck J (2019) Concussion Education Programs for High School Football Coaches: An Overview. J Pub Health Issue Pract 3: 136. https://doi.org/10.33790/jphip1100136.

Copyright: ( 2019 , This is an open-access article distributed under the terms of the Creative Commons Attribution License 4.0, which permits unrestricted use, distribution, and reproduction in any medium, provided the original author and source are credited.

\begin{abstract}
The increasing epidemic of concussion injuries in sports continues to attract significant attention throughout society. Leaders within the field of sports medicine have attempted to address athlete safety through educational programs. Although concussion education for athletic coaches is required by all states, individual learners should be considered when implementing these programs. Varying educational initiatives, lack of appropriate healthcare providers at athletic events, the difficulty recognizing the "invisible injury" by a diverse group of individuals, and the lack of enforcement of enacted laws are just a few obstacles that inhibit the concussion educational process required by legislation in all states. Appropriately delivered educational programs have the potential to improve management of sport-related concussion (SRC) at high school levels, providing a significant benefit to individuals across all societal levels. A comprehensive overview of commonly used concussion education programs provides a foundation of themes included in each program, while elucidating differences in program lengths, delivery methods, and targeted audiences. However, these concussion educational programs have been largely ineffective regarding injury reporting and participant safety. Researchers must study the effectiveness of the current programs through a lens of the five principles of knowledge transfer to make recognizable progress within this public health priority. A few recommendations to enhance the effectiveness of concussion educational programming include utilizing a dispersed delivery method to decrease the cognitive load and fatigue of participants, integrating reflective actions into modes of delivery for coaches, and employing reminder messages as cues to action. Schools would benefit from formally evaluating their current educational structure to ensure alignment with some of the recommendations.
\end{abstract}

Keywords: Sport-related concussion (SRC), Education, Knowledge Transfer, Liability

\section{Introduction}

Concussions continue to be a serious epidemic in youth and competitive sports. The incidence of sport-related concussions continues to rise, as approximately 1.6 million to 3.8 million sportrelated concussions occur each year [1-3]. In a 2017 national survey, $15.1 \%$, or approximately 2.5 million, of high school students reported having at least one concussion during the 12 months before the survey, and $6.0 \%$ reported two or more concussions [4]. Sport-related concussion (SRC) is defined as "a traumatic brain injury induced by biomechanical forces" that may occur with a direct blow or indirect force to the head, face, or neck [5]. Since SRCs may be difficult to recognize, head trauma can be extremely harmful and detrimental to the career of any athlete. Short term effects of repetitive head trauma and multiple concussions have revealed mixed results; whereas effects on long-term health remain under investigation [6]. Due to the continued concern of concussive injuries during athletic participation, increased attention and research have been provided to enhance proper recognition and management of SRC.

Leaders within the field of sports medicine have attempted to address athlete safety through educational programs provided to athletic administrators, coaches, and staff at many levels of competition. Concussion legislation, adopted throughout the United States between 2009 and 2014, contains a preseason education provision targeting various groups including coaches, athletes, and parents [7-8]. Despite these enacted laws, legislation efforts alone maybe insufficient in changing the sports culture of concussion management [9].

Detection and management of concussion injuries may be improved through enrichment of educational modules and improvement of communication among athletes, coaches, and medical professionals. Rivara et al. (2014) stated that $69 \%$ of the athletes continued to participate with concussion symptoms even after state law implementation [9]. The initial recognition of SRC by coaches, which requires immediate removal from activity, is vital because of the failure for athletes to always self-report concussion symptoms [10]. Coaches are integral in recognizing signs and symptoms of concussions to assist healthcare providers in removing the athlete from participation to further reduce the risks associated with continued participation after suffering a concussion [11]. Athlete safety may be enhanced allowing for fewer risks of long-term health effects if knowledge is retained by coaches after concussion education programs $[1,12]$. Since the coaches' knowledge retention will increase the safety practices in sports, parents may be more willing to allow youth to continue to participate in exercise and team activities that provide physical, mental, and social benefit.

Effective education can reduce potential lawsuits against coaches, schools, and athletic organizations that may present unwanted media attention and unnecessary legal fees. Lawsuits concerning concussion related injuries in sports targeting large scale organizations, including 
National Collegiate Athletic Association (NCAA), Pop Warner, and state athletic associations, remain on the rise [13]. Because of these developing litigation battles, organizations, such as the NCAA, have placed the responsibility of protecting the athlete on the membership schools and employees [13]. Organizations can influence prevention of harm from sport-related concussions at multiple levels through effective concussion education for coaches [8]. For example, coaches' education may include information regarding contact recommendations for practices (primary), recognition of signs and symptoms (secondary), and appropriate return to play strategies (tertiary) that reduce potential harm from concussion injuries.

\section{Barriers to Effective Concussion Education}

The effectiveness of coaches' education to enhance appropriate behaviors and attitudes toward concussion injuries is paramount [14]. According to Rivara et al. (2014), 40\% of coaches were not aware of athletes exhibiting concussion symptoms even after completing concussion education mandated by state laws [9]. Most coaches recognized a need for additional educational training when it came to managing concussions in their football programs. A study by Faure and Pemberton (2011) of 128 Idaho high school football coaches demonstrated that $94.2 \%$ of participants confirmed the risk of concussion in football did concern them, while $86.3 \%$ of these high school football coaches identified the responsibility to be able to recognize the signs and symptoms of concussions and to safely return an athlete to play [15]. In addition, $90 \%$ of youth hockey coaches rated knowledge about concussions as being highly important in their role as a coach [16]. Although research indicates that most high school and youth coaches accept their role with concussion recognition, there remains a disparity between educational programs and concussion awareness.

The inaccessibility of appropriate healthcare providers during sport activities further indicates the need for coaches to appropriately recognize concussion injuries to remain compliant with recent implementation of state laws. Certified athletic trainers are the most appropriate medical professional to provide care to high school athletes during participation [17]. Pryor et al. (2015) stated that 70\% of public high schools within the United States have access to a certified athletic trainer (AT), while only $37 \%$ of student-athletes have access to a full-time certified athletic trainer [18]. In a descriptive epidemiologic study by Esquivel, Haque, Keating, Marsh, and Lemos (2013), the authors found that ATs were not available at more than $75 \%$ of soccer and hockey practices in the state of Michigan [19]. ATs are often absent from practices and competitions at the youth level causing even greater responsibility for the coach to properly recognize concussive injuries [20]. The lack of availability of a certified athletic trainer during practices and competitions continues to inhibit progress with appropriate concussion management while placing the burden of responsibility on the coach. Organizations and schools must recognize the significant liability that may be present if a certified athletic trainer is not available at events and a coach is unable to recognize and properly manage a concussion injury.

No standardized method for training and educating coaches on concussion management currently exists $[6,21,22]$. Although online concussion education programs have been developed, schools and youth leagues still have options to develop their own educational materials without any medical supervision. Provvidenza and Johnston (2009) noted that it is critical to establish a consistent educational process for coaches who require education on concussion management [14]. Additionally, research indicated that athletic coaches often learn to become more aware of their behavior through some type of reflection, whether it be reflection in action, reflection on action, on retrospective reflection on action [14,23].

Assuring knowledge retention after online concussion education for high school coaches is challenging, but also paramount to address the epidemic with SRCs for youth athletes. Coaches embrace the significant role that they play throughout the concussion management process, as they inherently want to learn more about concussions to protect the athletes under their watch. Faure and Pemberton (2011) found that many Idaho high school head football coaches recognized their duty to determine when it was safe to allow a concussed athlete to return to activity, especially in the absence of an appropriate healthcare provider [15]. Although some coaches within the Faure and Pemberton (2011) study expressed hesitation referring suspected concussions to physicians, coaches shouldnever make return to play decisions, but only have the responsibility of removing the athlete from play. As mandated by most state laws, coaches should never return an athlete with a suspected concussion to play without an appropriate evaluation and return to play clearance by a qualified health care professional. Because of this responsibility, concussion educational programs for coaches must be designed to allow for early recognition and proper management of concussion injuries. As legislative requirements continue to mandate concussion educational initiatives, effective concussion educational programs for high school coaches should be identified to improve care of an injured athlete while reducing current preconceived misconceptions that still exist from prior mismanagement [15,24].

\section{State Law Challenges Impacting Concussion Legislation}

In May 2014, President Barack Obama held a summit on youth sport concussions at the White House where experts discussed the future of concussive injury and recovery, further validating the significant growth in injury awareness and its potential impact on public health [25]. Although being recognized as a public health priority is an essential first step for proper concussion management, many states face substantial challenges to the current legislation.

State concussion laws are initially increasing the awareness surrounding the concussion epidemic [7]. In a survey of 59 head football coaches and athletic trainers in the state of Washington, the results showed that one-half of respondents did not change their concussion management practices after law implementation although awareness of injury may have increased [26]. According to Lowrey (2015), the emphasis of current laws is on secondary prevention efforts after an injury has been sustained rather than focusing on primary prevention of these injuries, such as equipment advancement, practice modifications, or technological advancements [7]. The effectiveness of safety equipment, such as helmets and mouthpieces, used during sports participation continues to be investigated within the literature. Currently, the evidence does not support that helmets and mouthpieces reduce the incidence of concussions, but this protective equipmentmay reduce other serious injuries, such as skull fractures and injuries to the face and mouth [5,6]. Even though education may be utilized as a primary prevention technique, no framework has been developed to support this mandated aspect of the laws. These findings further show that legislation efforts alone will not suffice in changing the culture of concussion injury management in sports [9].

Moreover, lack of standardization of educational programming along with minimal enforcement of these state laws can lead to problems with effectiveness $[8,21,27,28]$. The content of concussion education programs differed greatly among several studies [25]. Many of the concussion laws include the discrepancy between uniformity "on the books" as compared to implementation procedures [7]. For example, many essential terms, including "youth", "qualified medical professional", and "education", within the laws go undefined leading to inconsistencies with implementation throughout various states and organizations. Variations exist among states in the specific educational requirements since each state can mandate a specific program or establish minimum standards for concussion education programs for key stakeholders, including coaches [29]. Due to the lack 
of specificity with content in the educational mandates with these state laws, significant differences may be noticed during the delivery of SRC education [8].

Besides the lack of standardization, the inability to enforce the law or punish non-compliant individuals remains a significant problem. One issue that troubles many of these enacted state mandates includes the inability to name an agency with authority to enforce the regulations [28]. Many of the responsibilities to track compliance have been assigned to third parties, such as school boards, athletic associations, or departments of health [21]. Kerr at al. (2014) explained only four laws designate specific punishments for non-compliance; whereas Harvey (2013) reveals that only five states implement evaluations to assess the efficacy of the coach education programs [21,27]. Many of the requirements within the laws, including educational mandates, are unable to be enforced to assure compliance because of the uncertainty of formal authority [28]. Although the substance of these laws does not vary much from the original law passed in the state of Washington, the lack of standardization and inability to mandate compliance remain significant challenges to ensuring consistent effective education for high school coaches.

\section{Sports Concussion Education Programs}

A comprehensive view of the commonly used concussion education modules around the country will be presented. The programs described include CDC's Heads Up: Concussion in Youth Sports, Think First, Sports Legacy Institute Community Educators (SLICE), Brain 101: The Concussion Playbook, Barrow Brain book, and Concussion Wise for Coaches. Themes that emphasize prevention, recognition, assessment, management, and return to play guidelines for concussions are common throughout most of these programs, but none of the programs have solid evidence that they are fully equipped for proper concussion education for coaches [30].

\section{Heads Up: Concussion in Youth Sports}

The CDC has developed fact sheets, handouts, and teaching guides that have become the model for sport-related concussion information and teaching tools [29]. This free toolkit includes an online video encompassing information on preventing, recognizing, and responding to concussions as well as concussion basics and return to play guidelines $[29,30]$. Coaches, parents, and health educators are targeted by this educational program, but the target audience does not include student-athletes because the developers rely on the previous mentioned groups to disseminate the information to the athletes [29]. The CDC has recently partnered with NFHS to produce a similar program for High School Sports entitled Concussion in Sports. The most recent version of this intervention takes approximately 45 minutes to complete all five modules and is commonly used by multiple states including Illinois, Iowa, Louisiana, Maryland, Massachusetts, Minnesota, Pennsylvania, Rhode Island, and Washington [30].

\section{Think First}

Think First is an educational tool founded in 1986 by the American Association of Neurological Surgeons and the Congress of Neurological Surgeons [29]. The creators of this 50-minute long program apply science and personal testimonies to promote safe behaviors and prepare individuals to prevent, recognize, and respond appropriately to concussions [29]. The developers of this concussion education program use health class presentations to educate all students on the prevention of brain and spinal cord injuries. Some of the objectives of this program include defining concussions, describing how they occur, reporting signs immediately, removing an individual from play, seeking medical attention, and following an appropriate treatment protocol [29]. The program developers also emphasize carefully monitoring a gradual return to daily activities, school, and sports.

\section{Sports Legacy Institute Community Educators}

(SLICE): The Sports Legacy Institute Community Educators (SLICE) was designed to advance the study, treatment, and prevention of concussions in youth athletes [29]. The 1-hour age-appropriate interactive presentation delivered by medical and health-related students includes discussion, videos, audience demonstrations, and case studies of professional, collegiate, and high school athletes $[1,29]$. Designed for athletes at all levels, the authors of this program strive to answer what is a concussion, the significance of injury to individuals, and what can be done about the injury [29].

Bagley et al. (2012) performed a prospective cohort study of 636 students between the ages of 9-18 to analyze the effectiveness of the SLICE curriculum on recognition and appropriate responses to concussions [1]. The participants displayed significant improvements in concussion knowledge quiz mean scores between pre-presentation and post-presentation [1]. In addition, $34 \%$ of students passed prepresentation quizzes compared to an $80 \%$ post-presentation pass rate further supporting the effectiveness of this student educational intervention [1]. Improvement in student-athlete's knowledge after educational programming may help reduce the barrier of understanding the risk of continued participation after concussion injury. Even though one barrier may be mitigated through athlete education, other barriers may inhibit concussion self-reporting, including excessive competitiveness and viewing injuries as a weakness. Because these barriers are intertwined with the culture of sport, it is critical that coaches can initially recognize a concussion injury to efficiently remove an athlete from play if the injury goes unreported.

Brain 101: The Concussion Playbook: Brain 101: The Concussion Playbook, was created by the Oregon Center for Applied Science (ORCAS) in 2011 [11,30]. Formerly known as Athletic Concussion Training using Interactive Video Education (Active), this comprehensive 20-minute, web-based concussion management program includes four modules: recognition, response, return to play, and prevention[30]. Although modules are geared toward various individuals, this interactive e-learning program was originally designed to target coaches of youth ages 10-18 on effective sports concussion prevention and management practices [11,29]. This course also shows signs and symptoms of a concussion and indicates an appropriate plan to return the student athlete to school and sport [29]. The authors of this program try to reduce the risk of concussions in youth student athletes while also encouraging a unified community response [29].

Barrow Brain book: Barrow Brain book was launched on August 18, 2011 through the collaboration of many organizations as the most comprehensive educational program in the state of Arizona [29]. This e-learning program, which is based on the content and instructional design used by the CDC, is designed to help students prevent, recognize, and respond to concussions appropriately [29]. This program is unique, as the developers use social networks, videos, and computer-generated animations to deliver the content, while also including a concussion registry for injuries as part of their educational design [29].

Concussion Wise for Coaches: Concussion Wise ${ }^{\mathrm{TM}}$, developed by Sports Safety International, is an online concussion education course, in which the creators use an engaging, interactive format for athletic trainers, physicians, coaches, parents, and other health care providers [31]. This free, online, 30-minute course for coaches includes a pre-test and post-test while covering the prevention, preparation, response, and return to play for concussion injuries [31]. This company currently has course modules designed for the following states: Colorado, Connecticut, Delaware, Florida, Nebraska, New Jersey, New York, Pennsylvania, Tennessee, Utah, and Wisconsin [30]. 
In summary, a variety of educational modules have been developed to use throughout the United States to educate high school coaches on the appropriate management of SRCs. However, many of these programs have never assessed knowledge retention over time for their target audiences. Because of the multitude of educational programs, there remains a lack of consensus on what program should be implemented throughout the United States. There is a significant need to evaluate the effectiveness of current online concussion education programs and develop more effective population-specific educational modules [24,32].

\section{Discussion}

Education and training may positively impact a coach's comprehension of sports concussion prevention and management since coaches have demonstrated significantly more concussion knowledge than the public after educational encounters [11,33]. Although the Institute of Medicine (IOM) and National Research Council (NRC) stated that concussion education programs may be effective for improving concussion knowledge and awareness, these same educational programs have had minimal effect in reducing the common behavior by athletes of continued participation while being symptomatic [6,24]. Coaches' concussion education should never supplant the need for access to a qualified health care professional during sport participation. A severe shortage of health care professionals dealing with athletes at the secondary school level is problematic. Society must promptly address the dearth of athletic trainers at this level, as coaches should never manage this complex injury independent of a qualified health care professional.Faure and Pemberton (2011) stated that $76.7 \%$ of high school coaches in Idaho did not feel adequately trained in concussion management, and $94.2 \%$ of coaches said the risk of concussion in football concerned them[15]. Finally, in a cross-sectional study of 126 high school coaches, O'Donoghue et al. (2009) found coaches in public and private high schools in southeastern Virginia were eager and willing to learn more about concussions if the materials were accessible [10]. These studies indicated the need and expressed motivation of high school coaches to undergo additional effective concussion education courses.

Availability of resources may be different among coaching levels, types of leagues, and sports, although coaches share many of the same attributes. High school coaches had more resources available to them through associations, conferences, or workshops, while youth sports coaches received education from a lay population [10]. Researchers have uncovered some significant differences on the effectiveness of concussion education for coaches among different sports. Approximately one-third (37\%) of the hockey coaches indicated they had limited knowledge about concussions, while only 1 in 5 of these coaches rated themselves as having a high knowledge base of concussion symptoms [16]. Faure and Pemberton (2011) found high school coaches were likely to make a return to play decision at smaller schools [15]. Finally, Esquivel et al. (2013) also found a difference in concussion education among high school sports in Michigan, as education was well promoted in high school football, but education expansion was recommended specifically for soccer and hockey [19]. Although mandatory education has been implemented across various levels, leagues, and sports, inequities have continued to be elucidated throughout multiple studies.

Many studies have shown the need to further evaluate specific educational techniques used to educate coaches because of the seriousness of concussion injuries, the growing responsibilities of high school coaches, and the various delivery methods of educational programming. DePadilla et al. [4] identified the importance to create a culture of safety when managing concussions; to expand educational efforts for students, parents, and, coaches; and to identify programs and policies that prevent concussions [4]. The IOM and NRC (2015) recommended that groups develop, implement, and J Pub Health Issue Pract Volume 3. 2019. 136 evaluate the effectiveness of specific educational techniques to increase knowledge about concussions in various groups of individuals [6]. Many authors recommended additional research to investigate the retention of knowledge obtained from educational programs on SRCs [10-11,27]. Evaluations are essential to enhance the effectiveness of concussion education programs while also increasing concussion reporting and reducing recurrent injuries. It is critical to identify whether changes in knowledge, attitude, selfefficacy and intention after educational concussion programs are maintained over time. Validation for efficacy of these programs must not be overlooked even though education alone may never be able to increase widespread awareness or normalize practice within concussion management. Researchers and investigators must study the effectiveness of the current programs to make recognizable progress within this public health priority.

Online education for sports concussions continues to become an attractive option compared to traditional face-to-face courses because of the advantage of flexible scheduling for learners [34]. High school coaches continue to be inundated with many classroom and athletic responsibilities due to frequent changes in the educational system. These multiple responsibilities may inhibit attendance at face-toface courses and encourage online education for these individuals. Furthermore, coaches in rural schools may not have the appropriate access to athletic trainers or concussion training centers for face-toface courses, which may prompt them to use an online concussion education option to meet legislative requirements. Although knowledge may not decay as rapidly as skill, the identification of the inability to retain knowledge is critical because the gap in knowledge retention may lead to the inability to apply the necessary skills during an emergency [35].

Despite the advantages of an online education option, many other important features must be considered. First, messages must be structured appropriately to be perceived as intended by the sender [36]. Januário et al. (2013) stated that the receivers' ability to recall information depends on the extent of engagement with the delivered message [36].During many online educational courses, the receiver has limited opportunity for engagement with the message leading to a possible barrier during the encounter. Many educational workshops for high school coaches occur in a mass delivery format at the beginning of the school year. Even more problematic is that these training formats often include a significant amount of information on many topics unrelated to concussion injuries in sports, which may be even more harmful to knowledge retention for high school coaches. Furthermore, repeating information more frequently enhanced knowledge retention; whereas, students' motivation to participate influenced their attention during these activities [36]. Rehearsal, or mental review of information, serves a key role in retention of knowledge [37]. Implementation of a dispersed delivery method to improve long-term knowledge retention should be considered to decrease the cognitive load and fatigue impacting the participants [38]. All these factors must be thoroughly considered during the process when assessing the effectiveness of an online concussion education program to enhance knowledge retention for high school coaches.

Mode of delivery is one critical component that can enhance knowledge retention [14,39].In a cohort study of high school coaches in the state of Washington, Rivara et al. (2014) identified in-person concussion training as having the most proficient score on coach awareness for presence of concussion symptoms in athletes; whereas a video and quiz may have been less effective than other modes of delivery [9]. Provvidenza and Johnston (2009) found coaches use reflective processes more effectively throughout learning endeavors [14]. Concussion education programs should be easily accessible, currently updated, and administrative friendly to enhance knowledge retention of participants $[25,40]$.

JPHIP, an open access journal ISSN- 2581-7264 
Many schools rely on the passive dissemination of information through distribution of educational materials and electronic programs because of the time scheduling constraints and varying obligations of high school coaches. Although these methods of education may be the most convenient, these methods do not garner consistent evidence to support knowledge retention. Januário et al. (2013) stated that the receivers' ability to recall information depends on the extent of engagement with the delivered message [36]. Schools would benefit from formally evaluating their current educational structure to enhance motivation of participants through engagement and to ensure alignment with some of the following components.

It is critical to establish an independent educational process that uses techniques for specific groups required to receive concussion education [14]. The effectiveness of educational programs for a specific audience group should be considered since many groups have different knowledge sets and diverse experiences with concussive injuries [14,41]. The five essential principles that must be considered when developing these programs include identifying the following parameters: target audience, delivered message, deliverer of the message, method of message transfer, and impact of knowledge transfer $[14,39]$.

Creators of educational programs should individualize the course for each group. The CDC has developed fact sheets, handouts, and teaching guides that have become the model for sport-related concussion information and teaching tools [29]. The targeted audiences for these educational tools include coaches, parents, athletes, and sport officials. Despite individualized resources for various groups, only one video is currently used for online education for all audience groups. For example, coaches and officials are commonly on the field of play; whereas, administrators and parents may be in the stands as spectators. It may be more beneficial to create concussion education programs that target each specific group rather than having all the groups matriculate through the same course.

When considering the method of message transfer specific to coaches, Provvidenza and Johnston (2009) found coaches use reflective processes more effectively throughout learning endeavors [14]. The utilization of videos that allow coaches to reflect on proper and improper management of previous concussion injuries may encourage interaction between coaches. Furthermore, implementation of a dispersed delivery method to improve long-term knowledge retention should be considered to decrease the cognitive load and fatigue impacting the participants [38]. Program administrators can utilize reminder messages as cues to action to reinforce key components of proper SRC management procedures. The impact on knowledge transfer should involve consistent evaluative procedures to celebrate success and address identified concerns. Furthermore, establishing a consistent group of individuals to enforce legislative requirements and evaluate educational programming could be beneficial.

The creators of coaches' concussion education programs should also focus on addressing societal barriers associated with the athlete that coaches may encounter, including the culture within a team and an athlete's attitude toward injury reporting $[9,42]$. Furthermore, several coaches acknowledged the pressure to win or play from various sources, including parents, school administrators, and athletes themselves, had influenced their decision-making regarding concussion management [15]. Because of the burdens placed on coaches, an obvious conflict of interest is present when attempting to remove an athlete from play, which makes the required health care provider return-to-play clearance in many state laws an essential component for appropriate management. As coaches and medical professionals encounter pressure form a variety of sources, it will be important to elucidate and evaluate the pressures placed on coaches and staff members from parents, administrators, athletes, and fans when handling return to play for serious injuries.

\section{Conclusion}

Sport-related concussions continue to be a serious epidemic for youth participants. A comprehensive overview of commonly used concussion education programs throughout the country has been provided. Assuring knowledge retention after online concussion education for high school coaches is challenging but also paramount to address the epidemic with SRCs for youth athletes. Some barriers to effective concussion education for high school coaches include lack of access to an appropriate healthcare provider, lack of standardization of programs, and inability for responsible agencies to enforce enacted laws. Since inequities have continued to be elucidated across various levels, leagues, and sports, schools and organizations would benefit from formally evaluating their current educational structure while also addressing the dearth of sports medicine professionals present at practices and competitions. Knowledge transfer principles must be considered when developing and evaluating evidence-based concussion education programs for high school coaches.

Effective education can reduce potential lawsuits that may present unwanted media attention and unnecessary legal fees. The important and imminent need for effective concussion education to enhance knowledge retention in high school football coaches has clearly been established. A few recommendations to enhance the effectiveness of concussion educational programs include utilizing a dispersed delivery method, integrating reflective actions for coaches, and employing reminder messages throughout the year. Time is of the essence when implementing these recommendations for concussion education programs, as the improper management of concussions by coaches puts the health and welfare of each athlete at risk during sports participation.

\section{Acknowledgements}

The authors would like to recognize and acknowledge fellow colleagues, Dr. Tommie Church, Dr. Myra Lovett, and Dr. Paula Griswold, for their support and feedback throughout the literature review process.

Conflict of Interest (COI) Statement: The authors confirm that this article content does not have any conflicts of interest

\section{Reference}

1. Bagley AF, Daneshvar DH, Schanker BD, Zurakowski D, d'Hemecourt CA et al. (2012) Effectiveness of the SLICE program for youth concussion education. Clinical Journal of Sport Medicine: Official J Can Acad Sport Med 22: 385-389.

2. Langlois JA, Rutland-Brown W, Wald MM (2006) The epidemiology and impact of traumatic brain injury: A brief overview. J Head Trauma Rehabilitation, 21: 375-378.

3. Mitchko J, Huitric M, Sarmiento K, Hayes G, Pruzan M et al. (2007). CDC's Approach to Educating Coaches about SportsRelated Concussion. Am J Health Edu 38: 99-103.

4. DePadilla L, Miller GF, Jones SE, Peterson AB, Breiding MJ et al. (2018) Self-reported concussions from playing a sport or being physically active among high school students - United States, 2017. Morbidity and Mortality Weekly Report 67: 682-685.

5. McCrory P, Meeuwisse W, Dvorak J, Aubry M, Bailes J, et al. (2017) Consensus statement on concussion in sport-the 5(th) international conference on concussion in sport held in Berlin, October 2016. Brit J Sports Med.

6. Institute of Medicine (IOM), \& National Research Council (NRC) (2015) Sports-related concussions in youth: Improving the science, changing the culture. Military Medicine 180: 123-125.

7. Lowrey KM (2015) State laws addressing youth sports-related traumatic brain injury and the future of concussion law and policy. J Bus Technol Law 10: 61-72. 
8. Register-Mihalik J, Baugh C, Kroshus E, Kerr ZY, Valovich McLeod TC (2017) Multifactorial approach to sport-related concussion prevention and education: Application of the socioecological framework. J Athletic Train 52: 195-205.

9. Rivara FP, Schiff MA, Chrisman SP, Chung SK., Ellenbogen RG, et al. (2014) The effect of coach education on reporting of concussions among high school athletes after passage of a concussion law. Am J Sports Med 42: 1197-1203.

10. O’Donoghue EM, Onate JA, Van Lunen B, Peterson CL (2009) Assessment of high school coaches' knowledge of sport-related concussions. Athletic Training \& Sports Health Care: The J Pract Clinic 1: 120-132.

11. Glang A, Koester MC, Beaver S, Clay J, McLaughlin K (2010) Online Training in Sports Concussion for Youth Sports Coaches. Int J Sports Sci Coaching 5: 1-11.

12. White PE, Newton JD, Makdissi M, Sullivan SJ, Davis G (2014) Knowledge about sports-related concussion: Is the message getting through to coaches and trainers? Brit J Sports Med 48: 119-124.

13. Pachman S, Lamba A (2017) Legal aspects of concussion: The ever-evolving standard of care. J Athletic Training 52: 186-194.

14. Provvidenza CF, Johnston KM (2009) Knowledge transfer principles as applied to sport concussion education. Brit $\mathrm{J}$ Sports Med 43: 68-75.

15. Faure CE, Pemberton CLA (2011) An examination of Idaho high school football coaches' general understanding of concussion. Sport J 14: 1-9.

16. Mrazik M, Bawani F, Krol AL (2011) Sport-related concussions: knowledge translation among minor hockey coaches. Clin J Sport Med: Off J Can Acad Sport Med 21: 315-319.

17. Wallace J, Covassin T, Nogle S, Gould D, Kovan J (2017) Knowledge of concussion and reporting behaviors in high school athletes with or without Access to an athletic trainer. J Athletic Training 52: 228-235.

18. Pryor RR, Casa DJ, Vandermark LW, Stearns RL, Attanasio SM (2015) Athletic training services in public secondary schools: A benchmark study. J Athletic Train 50: 156-162.

19. Esquivel A, Haque S, Keating P, Marsh S, Lemos S (2013) Concussion management, education, and return-to-play policies in high schools: A survey of athletic directors, athletic trainers, and coaches. Sports Health: A Multidisciplinary Approach 5: 258-262.

20. Hainline B, Ellenbogen RG (2017) A perfect storm. Journal of Athletic Train 52: 157-159.

21. Harvey HH (2013). Reducing Traumatic Brain Injuries in Youth Sports: Youth Sports Traumatic Brain Injury State Laws, January 2009-December 2012. Am J Pub Health 103: 1249-1254.

22. Mrazik M, Dennison CR, Brooks BL, Yeates KO, Babul S (2015) A qualitative review of sports concussion education: prime time for evidence-based knowledge translation. Brit $\mathrm{J}$ Sports Med 49: 1548-1553.

23. Gilbert WG, Trudel P (2001) Learning to coach through experience: Reflection in model youth sport coaches. J Teach Physic Edu 21: 16-34.

24. Kroshus E, Garnett BR, Baugh CM, Calzo JP (2015) Social norms theory and concussion education. Health Edu Res 30: 1004-1013.

25. Caron JG, Bloom GA, Falcão WR, Sweet SN (2015) An examination of concussion education programmes: a scoping review methodology. Injury Prevention: J Int Soc Child Adolescent Injury Prevent 21: 301-308.
26. Murphy A, Kaufman MS, Molton I, Coppel DB, Benson J (2012) Concussion evaluation methods among Washington state high school football coaches and athletic trainers. PM \& R: J Injury Fun Rehabil 4: 419-426.

27. Kerr ZY, Register-Mihalik JK, Marshall SW, Evenson KR, Mihalik JP et al. (2014) Disclosure and non-disclosure of concussion and concussion symptoms in athletes: Review and application of the socio-ecological framework. Brain Injury 28: 1009-1021.

28. Lowrey KM, Morain SR (2014) State experiences implementing youth sports concussion laws: Challenges, successes, and lessons for evaluating impact. J Law Med Ethics 42: 290-296.

29. Williamson RW, Gerhardstein D, Cardenas J, Michael DB, Theodore N (2014) Concussion 101: the current state of concussion education programs. Neurosurgery 75: S131-135.

30. Simons BL (2013) The effectiveness of education interventions on coaching Education students' concussion knowledge, retention, and attitudes. (Unpublished doctoral dissertation). Georgia Southern University, Statesboro, GA.

31. Sports Safety International (SSI) (2014) ConcussionWise Courses.

32. Tator CH (2012) Sport concussion education and prevention. J Clin Psychol 6: 293-301.

33. Guilmette TJ, Malia LA, McQuiggan MD (2007) Concussion understanding and management among New England high school football coaches. Brain Injury 21: 1039-1047.

34. Girard JP, Yerby J, Floyd K (2016) Knowledge retention in capstone experiences: An analysis of online and face-to-face courses. Knowledge Management E-Learn 8: 528-539.

35. Popp JK, Berry DC (2016) Athletic training students demonstrate airway management skill decay, but retain knowledge over 6 months. Athletic Train Edu J 11: 173-180.

36. Januário NMS, Rosado AF, Mesquita I (2013) Variables affecting athletes' retention of coaches' feedback. Perceptual \& Motor Skills 136: 389-401.

37. Schunk DH (2012) Learning theories: An educational perspective. Boston: Pearson.

38. Raman M, McLaughlin K, Violato C, Rostom A, Allard JP, (2010) Teaching in small portions dispersed over time enhances long-term knowledge retention. Medical Teacher 32: 250-255.

39. Reardon R, Lavis J, Gibson J (2006) From research to practice: a knowledge transfer planning guide. Institute for Work and Health.

40. Stewart C (2006) Coach Education Online: The Montana Model. J Phy Edu Recrea Dance (JOPERD) 77: 34-36.

41. Provvidenza C, Engebretsen L, Tator C, Kissick J, McCrory $P$ et al. (2013) From consensus to action: Knowledge transfer, education and influencing policy on sports concussion. Brit $\mathbf{J}$ Sports Med 47: 332-338.

42. Sarmiento K, Mitchko J, Klein C, Wong S (2010) Evaluation of the Centers for Disease Control and Prevention's concussion initiative for high school coaches: "Heads Up: Concussion in High School Sports. J School Health 80: 112-118. 